\title{
The Minimum Detectable Damage as an Optimization Criterion for Performance-based Sensor Placement
}

\author{
Alexandler Mendler ${ }^{1}$, Michael Döhler ${ }^{2}$, Carlos E. Ventura ${ }^{1}$, and Laurent Mevel ${ }^{2}$ \\ ${ }^{1}$ Dept. of Civil Engineering, University of British Columbia, Vancouver BC, V6T 1 Z4 Canada (alexander.mendler@ubc.ca, ventura@civil.ubc.ca) \\ 2 Univ. Rennes, Inria, Ifsttar COSYS/SII, 35042 Rennes, France (michael.doehler@inria.fr, laurent.mevel@inria.fr)
}

\begin{abstract}
Selecting the right sensor locations is pivotal for the effectiveness of vibration-based damage detection as part of structural monitoring under unknown excitation. Traditional optimization criteria aim to increase the signal-to-noise ratio and information content carried by ambient vibrations or to identify modal parameters with minimal uncertainty and optimal mode distinguishability. None of the existing criteria appear to consider a relative decrease in material strength, although this is the decisive quantity for structural design, structural health, and thus safety. This paper fills this gap for stochastic subspace-based damage diagnosis methods. It introduces an optimization criterion that ensures that a minimum damage extent can be detected in each structural component while maintaining a probability of detection that meets the national design standards. The presented strategy helps to find an acceptable number of sensors as well as their optimal layout. Further advantages are that the optimization can be done based on the simulated vibration data from the healthy structure and that the criterion can be tuned so the resulting sensor layout becomes more sensitive to damage hotpots.
\end{abstract}

Keywords: Output-only, stochastic subspace-based damage detection, minimum measurement duration, Fisher information.

\section{INTRODUCTION}

There is an increasing demand in developing damage detection and localization methods for structural components of safetycritical infrastructure, e.g. lifeline bridges in areas of high seismic hazards [12]. An important part of the efficiency of the damage diagnosis is the sensor placement strategy. Optimal sensor placement has been subject to extensive research in both the engineering and the automatic control community, see $[14,7,16]$ and $[16]$. Recent literature reviews conclude that the existing optimization criteria suffice to pre-condition the signal with respect to the signal-noise ratio and the quality and uncertainty of the modal identification, but also outline that there is a demand for new, method-specific performance criteria that directly target the detectability of damage [19]. This paper proposes the measurement duration $\mathrm{T}$ [s] as a performance criterion for damage detectability and is written for stochastic subspace-based damaged detection (SSDD) $[6,3,10]$. It continues the line of work of previous authors $[4,9]$ that employed the Fisher information as a measure for the information content of ambient vibrations with respect to characteristic system parameters. This is equivalent to increasing the probability of detection and decreasing the false-alarms. It combines the approach with a formula for the minimum detectable damage, [13] which is used to interpret the Fisher information as a minimum measurement duration that is necessary to achieve a desired probability of detection. This paper is organized as follows: Section 2 recaps how a damage-sensitive criterion can be formed in the subspace of covariance functions, Section 3 derives the formula for the minimum measurement duration and Section 4 and 5 optimizes the sensor placement on a pin-supported beam and discuss the results.

\section{BACKGROUND}

\subsection{Stochastic System Model}

Let the vibration signal be a realization of a linear and time-invariant (LTI) dynamic system with $m$ degrees of freedom

$$
\mathcal{M} \ddot{z}(t)+\mathcal{C} \dot{z}(t)+\mathcal{K} z(t)=v(t) \text { where } \mathcal{K}=f\left(E_{1}, . ., E_{H}\right)
$$

where $z(t)$ is the displacement under random disturbances $v(t)$ in continuous-time and $\mathcal{M}, \mathcal{C}$ and $\mathcal{K} \in \mathbb{R}^{m \times m}$ are the mass, damping and stiffness matrices. This papers will assume that structural damage is limited to changes in the stiffness matrix, which are a function of the stiffness of each structural component, i.e. the modulus of elasticity $E_{h}[\mathrm{MPa}]$ for $h \in[1, \ldots, H]$. 


\subsection{Subspace-based Residual}

The residual is formed based on measurement data in discrete-time, i.e. displacement, velocity, or acceleration data at some of the degrees of freedom of (1). For each time instant $k$, the data is stored in the measurement vector $y_{k} \in \mathbb{R}^{r}$ where $r$ is the number of sensors. To analyze the similarity of the wave pattern at different time instances and sensor locations, the output covariances $R_{i}=E\left(y_{k+i} y_{k}^{T}\right)$ are estimated by $\hat{R}_{i}=\frac{1}{N-i} \sum_{k=1}^{N-i} y_{k+i} y_{k}^{T}$ where $i$ is the time lag. Output covariances can be interpreted as impulse response functions that model the system output under random excitation for $i \geq 1$, see [11,17]. To capture the (hidden) vibration model of the dynamic system, the covariances are arranged in an (output) block Hankel matrix.

$$
\hat{\mathcal{H}}_{p+1, q}=\left[\begin{array}{cccc}
\hat{R}_{1} & \hat{R}_{1} & \ldots & \hat{R}_{q} \\
\hat{R}_{2} & \hat{R}_{1} & \ldots & \hat{R}_{q+1} \\
\vdots & \vdots & \ddots & \vdots \\
\hat{R}_{p+1} & \hat{R}_{p+2} & \ldots & \hat{R}_{p+q}
\end{array}\right]=\left[\begin{array}{ll}
\hat{S}_{1} & \hat{S}_{0}
\end{array}\right]\left[\begin{array}{cc}
\hat{V}_{1} & 0 \\
0 & \hat{V}_{0}
\end{array}\right]\left[\begin{array}{c}
\hat{D}_{1}^{T} \\
\hat{D}_{0}^{T}
\end{array}\right]
$$

The time lag parameters $p$ and $q$ are set large enough so all modes (in particular low-vibration modes) are observable with $q r>2 m$ and typically $p+1=q$. Since modes merely have to be observable but not identifiable, considerably shorter time lags are required for damage detection in comparison to modal identification. Applying a singular value decomposition to the block Hankel matrix leads to several desirable effects. Firstly, more dominant trends, such as structural vibrations are stored in the upper part $\hat{S}_{1} \hat{V}_{1} \hat{D}_{1}^{T}$ and the lower part models noise, so $\hat{V}_{0} \approx 0$. Secondly, the singular vectors are orthogonal to one another and multiplying orthogonal vectors yields zero. That means that pre-multiplying the left null space (i.e. the vector space $\hat{S}_{0}$ corresponding to the singular values in $\hat{V}_{0}$ ) of the block Hankel matrix in the reference state eliminates all information related to structural vibrations in the reference state

$$
\varepsilon^{0}=\operatorname{vec}\left(\hat{S}_{0}^{T} \hat{\mathcal{H}}_{p+1, q}^{0}\right) \approx 0 .
$$

This approximation becomes exact if the measurement are sufficiently long $(N \rightarrow \infty)$ because then the estimated covariances approximate the theoretical one $\hat{R}_{i} \rightarrow R_{i}$ and so do the block Hankel matrix $\hat{\mathcal{H}}_{p+1, q} \rightarrow \mathcal{H}$ and the null space $\hat{S}_{0} \rightarrow S_{0}$. Using the central limit theorem, a Gaussian residual vector can be formulated with respect to the sample size $N$ [6],

$$
\hat{\zeta}=\sqrt{N} \varepsilon^{0} \sim \begin{cases}\mathcal{N}(0, \Sigma) & \text { (healthy) } \\ \mathcal{N}(\mu, \Sigma) & \text { (damaged })\end{cases}
$$

In other words, the residual vector $\hat{\zeta}$ follows a Gaussian distribution with mean value zero and covariance $\Sigma$ if both matrices $\hat{S}_{0}$ and $\hat{\mathcal{H}}_{p+1, q}$ are calculated based on the same healthy structure. Damage, on the other hand, manifests itself in a deviation from the zero mean condition by $\mu$.

\subsection{Link to Finite Element Model}

Let the structural system be characterized by $H$ health parameters. These parameters could, for example, be the stiffness and mass values of different structural components, which can be taken from an equivalent finite element (FE) model and have a direct relation to the system matrices $\mathcal{M}$ or $\mathcal{K}$ from Eq. (1). Since damage is assumed to exclusively affect the stiffness parameters, i.e. the material's modulus of elasticity, the structure can be characterized through the parameter vector

$$
\theta=\left[E_{1} \cdots E_{H}\right]^{T},
$$

where $\theta \in \mathbb{R}^{H}$. The superscript " 0 " indicates that the parameter vector is in its reference state. The Gaussian residual from Eq. (4) is purely data-driven and statistical in nature but its mean value vector can be linked to the physical parameters change in Eq. (5). This process is called parametrization. The deviation in the mean residual is replaced by $\mu=\mathcal{J} \delta$, where $\delta$ is the statistical change vector and $\mathcal{I}=\frac{\partial}{\partial \theta^{0}} E\left(\varepsilon^{0}\right)$ is the sensitivity (or Jacobian) matrix of the mean residual with respect to the chosen parametrization. The statistical change vector is a central element, as it transforms the change detection problem from the statistical domain (and monitoring the mean residual) into the physical domain of monitoring structural parameters, where [6]

$$
\delta=\sqrt{N}\left(\theta-\theta^{0}\right) \text {. }
$$

In our case, the physical problem is to detect a decrease in the material stiffness $E$, which can be linked to a change in the mean residual through three consecutively performed first-order sensitivity analyses combined through the chain rule

$$
\mathcal{I}=\mathcal{I}\left(\varepsilon^{0}, \theta_{d}^{0}\right) \cdot \mathcal{I}\left(\theta_{d}^{0}, \theta_{c}^{0}\right) \cdot \mathcal{I}\left(\theta_{c}^{0}, \theta^{0}\right) .
$$

The first term links the mean residual to the modal parameters in discrete-time in $\theta_{d}^{0}$, the second term links the modal parameter in discrete-time to the modal parameters of the FE model in continuous-time in $\theta_{c}^{0}$, and the third one links the modal parameters of the FE model to its structural parameters in $\theta$. More information on this can be found in the literature, see [1, 3, 5]. 


\section{METHOD}

\subsection{Minimum Detectable Damage}

To discriminate the healthy from the damaged state, it has to be tested whether the residual vector is more likely to be the realization of the damaged rather than the healthy structure, see Eq. (4). This can be done through statistical hypothesis tests, e.g. the general likelihood ratio (GLR), see [2], where the null hypothesis $\theta=\theta^{0}$ is tested against the alternate hypothesis $\theta=\theta^{0}+\delta / \sqrt{N}$ from Eq. (6). It can be shown, [3], that the GLR unfolds to

$$
t=\hat{\zeta}^{T} \Sigma^{-1} g\left(g^{T} \Sigma^{-1} g\right)^{-1} g^{T} \Sigma^{-1} \hat{\zeta} \sim \begin{cases}\chi^{2}(v, 0) & \text { (healthy) } \\ \chi^{2}(v, \lambda) & \text { (damaged })\end{cases}
$$

Since the residual with well-defined Gaussian properties is squared up, the test statistic follows a $\chi^{2}$-distribution with $v$ degrees of freedom and non-centrality $\lambda=\delta^{T} F \delta$, [3], where $F \in \mathbb{R}^{H \times H}$ is the Fisher information matrix

$$
F=g^{T} \Sigma^{-1} \mathfrak{g} .
$$

The number of degrees of freedom $v$ of the $\chi^{2}$-distribution is-in the simplest case-equal to the number of structural parameters $H$. This is only true if the Jacobian matrix is of full rank, which is assumed in this paper. Otherwise, it is given by

$$
\mathrm{v}=\operatorname{rank}(F) .
$$

The non-centrality is the distance between the healthy and damaged stated probability density function (PDF) with $\lambda=0$ and $\lambda>0$, respectively, see Fig. 1 . To ensure a sufficient degree of separation between the states, the desired non-centrality can be characterized by a minimum value for any parameter $h$,

$$
\lambda_{h}=F_{h h} \delta_{h}^{2} \geq \lambda_{\min }=\text { const. }
$$

This is equivalent to prescribing a minimum probability of detection (POD) in each of the $H$ health parameters, see Fig. (1). The non-centrality $\lambda_{\min }$ could, for instance, be calculated based on the maximum allowable false-alarm rate $\alpha$ and false-positive rate $\beta$ because both PDFs are mathematically well-defined, see Fig, 1, but more on this follows in the subsequent section. Solving Eq. (11) for $\delta_{h}$ and plugging it into Eq. (6) results in a formula for the minimum parameter change that can be detected reliably, [13].

$$
\theta_{h}-\theta_{h}^{0} \approx \sqrt{\frac{\lambda_{\min }}{N \cdot F_{h h}}}
$$

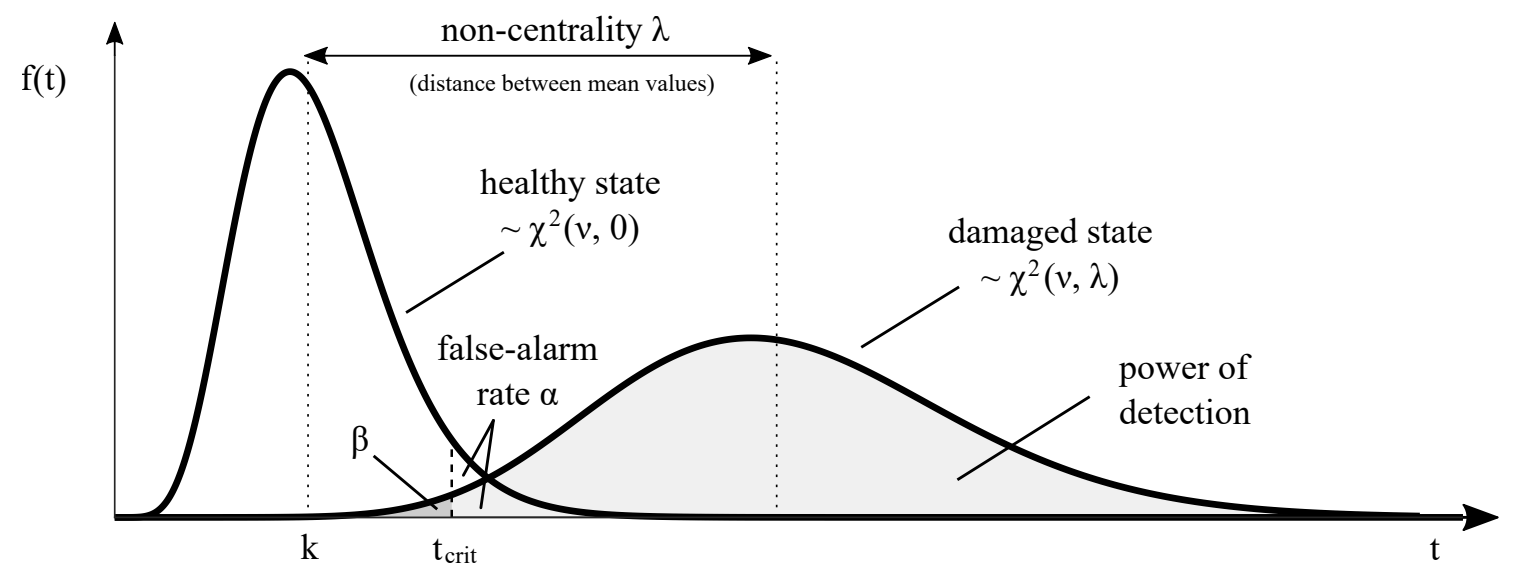

Figure 1: Statistical distribution of the test statistic from Eq. (8) in the healthy and damaged state, with reference to Eq. (13). 


\subsection{Minimum Reliability}

The $\chi^{2}$-distribution in see Fig. 1 is a function of two variables, i.e. the number of degrees of freedom $v$ and the non-centrality $\lambda$, which is zero in the healthy state. The probability density functions (PDF) are defined as

$$
f_{\chi^{2}(v, 0)}(t)=\frac{1}{2^{v / 2} \Gamma(v / 2)} x^{v / 2-1} e^{-t / 2} \text { (healthy), } \quad f_{\chi^{2}(v, \lambda)}(t)=\sum_{n=0}^{\infty} e^{-\frac{\lambda}{2}} \frac{(\lambda / 2)^{n}}{n !} f_{\chi^{2}(v+2 n, 0)}(t) \text { (damaged) }
$$

where $\Gamma(\gamma)$ is the Gamma function, satisfying $\Gamma(\gamma)=(\gamma-1)$ ! for integer values of $\gamma$. The healthy state is uniquely defined with $\lambda=0$, because the number of degrees of freedom $v$ is a known constant that depends on the monitoring application, see Eq. (10). The minimum non-centrality $\lambda_{\min }$ is a user-defined value and, as explained in this section, can be set by requiring a minimum reliability regarding the damage diagnosis result.

The false-alarm rate $\alpha$ is the probability that a false-alarm occurs, i.e. it quantifies how often the test diagnoses a healthy structure as damaged. Based on the acceptable false-alarm rate, a safety threshold value can be defined that discriminates the damaged from the healthy structure, see $t_{c r i t}$ in Fig. 1. Our recommendation for civil engineering structures is to set the safety threshold corresponding to a false-alarm rate of $\alpha \in[0.3 \%, 5 \%]$, or lower. In that case, one out of 20 - 333 tests diagnoses a healthy structure as damaged.

$$
\alpha=1-\int_{0}^{t_{c r i t}} f_{\chi^{2}(v, 0)}(t) d t
$$

The false-positive rate $\beta$ quantifies how often the test diagnoses the structure as healthy although damage is present, see Fig. 1. The consequences of setting the false-positive rate $\beta$ too high can be fatal, as small damage to key components may cause the collapse of the structure and put human lives at risk. In other words, the false-positive rate is adjusted with respect to the damage consequences and the acceptable level of risk. For load-bearing components, we recommend choosing this value according to the national safety concept. In Canada and the U.S.A., the reliability index for assessment is prescribed as 3.25 and 2.5, which is equivalent to a false-negative rate of $\beta=0.06 \%$ and $\beta=0.6 \%$, respectively. The Eurocode does not define a reliability index for assessment, but the ISO norm requires a reliability index of 4.7 , so $\beta=10^{-6}$ [18].

$$
\beta=\int_{0}^{t_{c r i t}} f_{\chi^{2}(v, \lambda)}(t) d t
$$

The minimum non-centrality can be determined by sliding the theoretical distribution for the damaged state to the right until the $\beta$-th quantile aligns with the safety threshold, see Eq. (13) and Fig. 1. The only unknown parameter in the curve-fitting process is the minimum non-centrality $\lambda_{\min }$ which can be solved for through numerical iteration. The non-centrality is the distance between the mean values, and the mean value of the healthy state PDF is $v$.

$$
\lambda_{\min }=\mu\left(f_{\chi^{2}(v, \lambda)}\right)-v
$$

\subsection{Minimum Measurement Duration}

The basic formula from Eq. (12) can be rearranged and solved for the measurement duration $T=N / f_{s}$. Moreover, the parameter change can be normalized through the reference parameter to obtain an expression for a relative parameter change $\Delta_{h}=$ $\left(\theta_{h}^{0}-\theta_{h}\right) / \theta_{h}^{0}$. The resulting equation estimates the minimum measurement duration required to diagnose the user-defined (relative) damage level, e.g. $\Delta_{h}=5 \%$, in each stiffness parameter.

$$
T_{h}(c) \approx \frac{\lambda_{\min }}{f_{s} F_{h h}}\left(\frac{1}{\Delta_{h} \theta_{h}^{0}}\right)^{2}[s]
$$

If the sampling frequency $f_{s}$ and the minimum non-centrality $\lambda_{\min }$ are fixed, the measurement duration depends on the magnitude of the health parameter in the healthy state $\theta_{h}^{0}$, the desired minimum detectable damage $\Delta_{h}$, and the main diagonal of the Fisher information $F_{h h}$, which depends on the sensors configuration $c$. To indicate this dependency, the bracket term is added to the measurement duration $T_{h}(c)$. If the desired damage extent is to be detected in each parameter, the decisive parameter is the one with the longest measurement duration. In this sense, the performance criterion for sensor placement optimization writes

$$
T_{\max }(c)=\max _{h}\left\{T_{1}, \cdots, T_{H}\right\} .
$$

The higher the Fisher information, the higher the sensitivity towards small damages, see Eq. (9), and the shorter the maximum measurement duration. The optimization goal for sensor placement is, therefore, to find the placement that minimizes the maximum measurement duration.

$$
\min _{c} f=T_{\max }(c)
$$




\section{NUMERICAL APPLICATION}

For proof of concept, the sensors placement strategy is applied to a pin-supported beam discretized into 18 elements and nine materials. The overall goal is to find an acceptable number of sensors as well as the optimal sensor placement that allows detecting a $\Delta=5 \%$ stiffness decrease in any material. The minimum reliability is defined through the false-alarm rate of $\alpha=5 \%$ and the false-positive rate of $\beta=0.6 \%$

HSS $152 \times 51 \times 4.78 \mathrm{~mm} \quad$ Sym.

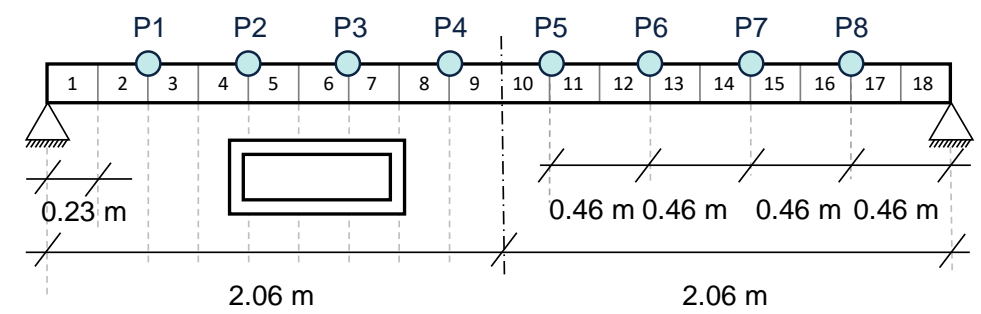

Figure 2: Pin-supported hollow structural steel (HSS) beam with possible sensor locations P1-P8.

\subsection{Pin-supported HSS Beam}

The structure under consideration is a $4.11 \mathrm{~m}$-long pin-supported beam with a hollow structural steel section, HSS $152 \times 51 \times 4.78$ $\mathrm{mm}$. The 3-D beam model is divided into 18 finite beam elements with a length of $22.8 \mathrm{~cm}$, see Fig. 2, where two consecutive elements are assigned to the same material (or health parameter) with a modulus of elasticity of $E_{h}=200.000 \mathrm{MPa}$ and $h \in$ $[1, \ldots, 9]$. The first four vertical modes of vibration are used to screen the beam for structural damage with natural frequencies of $8.97 \mathrm{~Hz}, 35.8 \mathrm{~Hz}, 80.3 \mathrm{~Hz}$, and $142 \mathrm{~Hz}$, a modal damping ratio of $1 \%$ critical damping, and the mode shapes visualized in Fig. 3. The system input is modelled as white noise and applied at each of the 104 degrees of freedom. To simulate measurement noise, uniformly distributed noise was added to the output with a magnitude corresponding to $5 \%$ of the output variance.

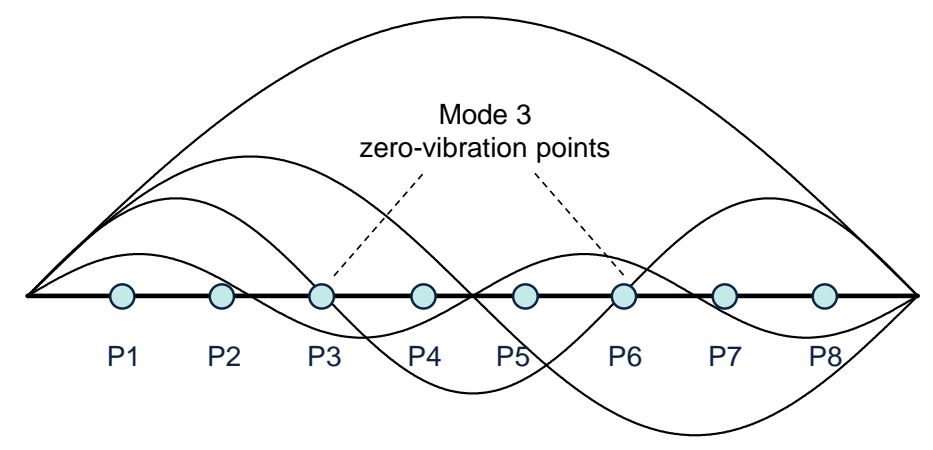

Figure 3: Zero-vibration points among the sensor locations P1-P8.

The required input for the sensor placement optimization is the vibration data from the healthy structure at all candidate locations. Hence, one transient analysis is run while recording the velocity in the vertical direction at eight selected locations, see Fig. 2. The measurement time for the simulation is set to $60 \mathrm{~min}$ and the sampling frequency for the simulation is set to $1024 \mathrm{~Hz}$. The resulting measurement matrix $Y\left(\theta_{0}\right)$ is handed over to the sensor placement optimization algorithm, it contains $r$ columns and $N$ rows where $r$ and $N$ are the number of sensors and samples, respectively.

$$
Y\left(\theta_{0}\right)=\left[\begin{array}{cccc}
y_{1,1} & y_{1,2} & \cdots & y_{1, r} \\
\vdots & \vdots & \ddots & \vdots \\
y_{N, 1} & y_{N, 2} & \cdots & y_{N, r}
\end{array}\right]
$$




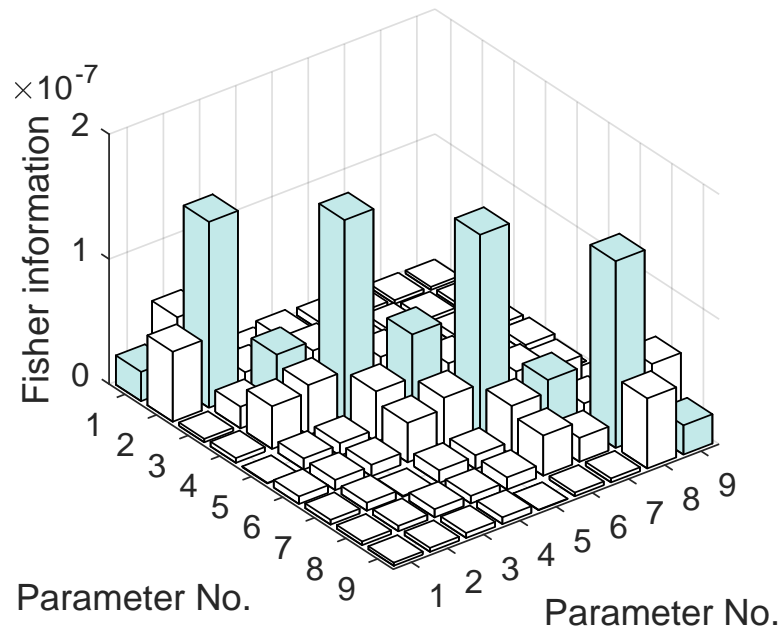

Figure 4: Fisher information for nine monitoring parameters and a typical sensor layout.

\subsection{Measurement Duration for a Fixed Sensor Configuration}

In this section, it is explained how the minimum measurement duration can be calculated for a fixed sensor configuration, for example, a single sensor at location P2. First, all input parameters are determined to calculate the performance criterion for the sensor placement, see Eq. (17). The minimum reliability requirements translate into a minimum non-centrality of $\lambda=33.7$, which corresponds to the minimum distance between the mean of the healthy and damaged stated PDF, see Fig. 1. The resampling frequency $f_{s}$ is fixed sufficiently high to capture all modes of vibration, so $f_{s}=360 \mathrm{~Hz}$, and the structural parameter to be monitored is the modulus of elasticity between each sensor, so $\theta_{h}=200,000 \mathrm{MPa}$ with $h \in[1, \ldots, 9]$. The last input quantity is the Fisher information, which describes the sensitivity of the Gaussian residual towards changes in the structural parameters, scaled by the residual's (co-)variance. It is the only quantity that is estimated based on vibration data, see Fig. 4 as well as Eq. (7) and (9). The main diagonal of the Fisher information can be interpreted as a minimum measurement duration in each parameter using Eq. (17), and the results are visualized in Fig. 5 (a). On close inspection of the bar plot ins this figure, it can be observed that the minimum measurement duration is almost symmetrical despite the asymmetrical sensor arrangement. Moreover, the measurement duration is the longest for parameters near low-vibration points, i.e. near the pin supports (with the decisive measurement time of $T_{\max }=101 \mathrm{~s}$ ) and at mid-span, where Mode 2 and 4 exhibit low vibration points, cf. Fig. 3. More generally, the distribution of the measurement duration per element depends on the modes of vibration that are used to screen the structure for damage. For demonstration, a second sensor layout with two sensors at position P1 and P8 is shown in Fig. 5 (b). Due to the increased number of sensors, the maximum measurement duration $T_{\max }$ decreases from $101 \mathrm{~s}$ to $56 \mathrm{~s}$.

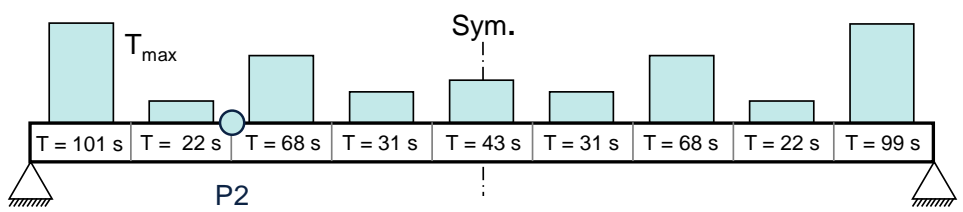

(a)

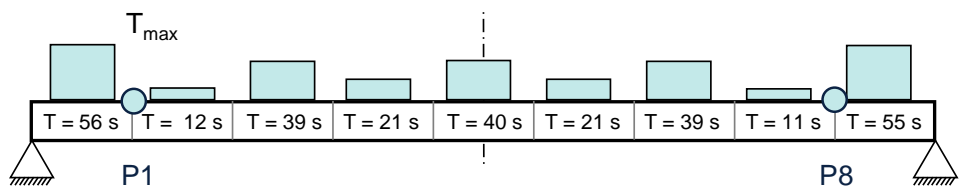

(b)

Figure 5: Minimum measurement duration $T_{h}$ from Eq. (17) (a) for one sensors at position P2, and (b) for two sensors at P1 and P8. 


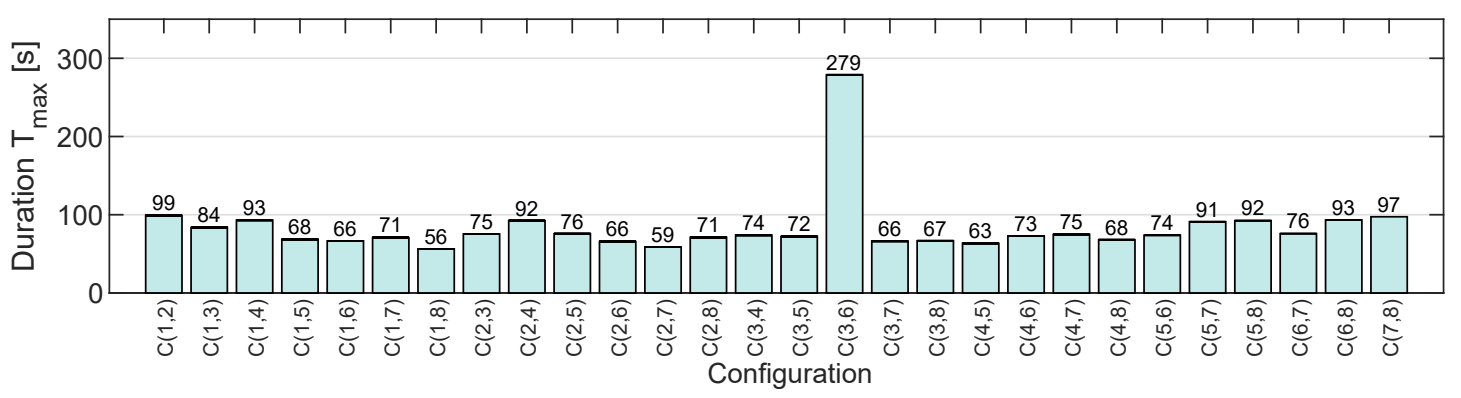

Figure 6: Maximum measurement durations $T_{\max }$ from Eq. (18) for all sensor configurations with two sensors.

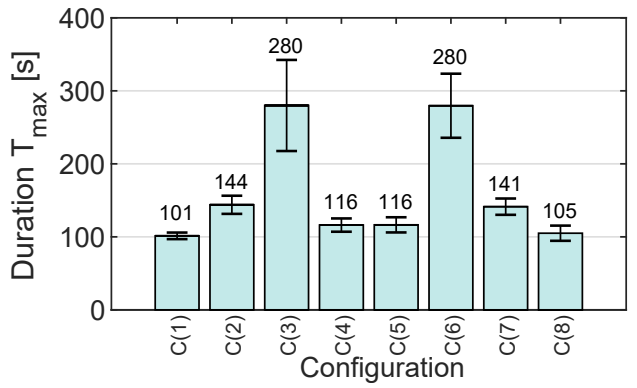

Figure 7: Maximum measurement durations $T_{\max }$ from Eq. (18) and its standard deviation $\sigma$ for one sensor.

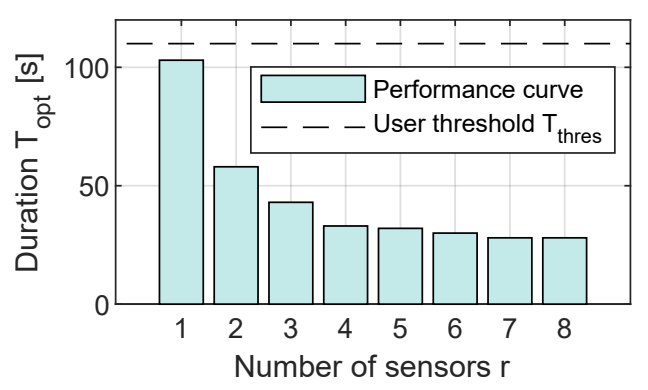

Figure 8: Optimal measurement time $T_{\text {opt }}$ from Eq. (22) for a varying number of sensors

\subsection{Optimal Sensor Placement for a Fixed Number of Sensors}

In this section, the sensor placement strategy for a fixed number of sensors with varying sensor location is outlined. The total number of sensor configurations is

$$
N_{c}=\frac{r_{p o s} !}{\left(r_{p o s}-r\right) ! r !}
$$

where $r_{p o s}$ is the number of possible sensor locations and $r$ is the number of sensors. For each sensor configuration, the maximum measurement time is evaluated using the approach that was described in the preceding section. Since the measurements at all sensor locations are available and stored in the measurement matrix from Eq. (20), the only thing left to do is to extract the respective columns, re-estimate the Fisher information, see Fig. 4, translate it into the minimum measurement duration, and find the decisive measurement duration among all elements as the one with the longest measurement duration. Ultimately, all configuration can be compared in an evaluation chart.

The evaluation chart is shown for the simplest case with only one sensor and $N_{c}=8$ possible sensors configurations, see Fig. 7. The optimal measurement duration is the one with the shortest maximum measurement duration, i.e. configuration number one or eight (symmetry) with $T_{\max }=101 \mathrm{~s}$. In contrast, the worst sensors configurations are configuration $\mathrm{C}(3)$ and $\mathrm{C}(6)$ with sensors at P3 and P6 and a measurement duration of $T_{\max }=240 \mathrm{~s}$. The sensor locations coincide with vibration nodes (zero-vibration points) of Mode 3, see Fig. 3. Consequently, Mode 3 is non-observable and does not contribute to the Fisher information which, according to Eq. (17), leads to a higher measurement duration. Non-observable modes should be treated with caution. Since the null space $S_{0}$ is estimated based on data, see Eq. (2), it is blind to Mode 3 . Reducing the stiffness in any health parameter (apart from $E_{5}$ at mid-span) introduces a non-symmetry in the system, which may cause the zero-vibration point of Mode 3 to shift and the mode to become observable. However, the null space is not orthogonal to Mode 3 , meaning the residual will not have the expected statistical properties. Moreover, zero-vibration modes can cause linear dependencies in the column space of the Jacobian matrix, which falsifies the predictions of the minimum detectable damage. The latter can be avoided if zero-vibration modes are removed from the analysis, e.g. by overwriting the corresponding entries in the Jacobian matrix with zero entries. Since this does not remedy the problem regarding the missing modes in the null space, it is recommended to exclude sensor configurations with non-observable modes from the optimization scheme. For the sake of example, the optimization scheme is also shown for sensor configurations with two sensors, see Fig. 6. Configuration $\mathrm{C}(3,6)$ has the longest measurement duration because the sensors are placed at P3 and P6, i.e. the zero-vibration points of Mode 3 . The best 
sensors configuration is $\mathrm{C}(1,8)$ with a measurement duration of $T=56 \mathrm{~s}$. Note that the best sensors configurations for one and two sensors were already discussed in the previous section, see Fig. 5.

\subsection{Finding an Acceptable Number of Sensors}

This section explains how to find an acceptable number of sensors. The main idea is to repeatedly evaluate the optimal sensor configuration for a fixed number of sensors, as explained in the previous section. For the problem at hand, there are eight sensors locations, and thus, we could use between one and eight sensors, so $r \in[1, \ldots, 8]$. As a result, we obtain the optimal sensor configuration for each number of sensors $r$ as well as the corresponding optimal measurement duration.

$$
T_{\text {opt }}(r)=\min _{c}\left\{T_{\max }(c)\right\}<T_{\text {thres }}
$$

To find an acceptable number of sensors, the optimal measurement duration can be plotted over the number of sensors, see Fig. 8. Now, the maximum allowable measurement duration $T_{\text {thres }}$ should be defined (based on the application) and the optimal sensor configuration can be selected as the one with the next-lowest measurement time.

\subsection{Validating the Minimum Detectable Damage}

The overall goal was to find the optimal sensor configuration and an acceptable number of sensors to detect a $5 \%$ stiffness decrease in any one beam element. For the sake of example, the optimal sensor configuration with one single sensor is chosen, because the measurement duration of $T=101 \mathrm{~s}$ is demmed acceptable. To validate the effectiveness of the obtained sensor layout, the stiffness in the decisive element is decreased by $5 \%$. Then, 100 new data sets with a duration of $T=101 \mathrm{~s}$ are generated in the damaged state, the statistical test is applied to each record, and the empirical damaged state histogram is compared to the theoretical distribution. The measure used for evaluating the accuracy of the predicted minimum measurement duration is the probability of detection, i.e. the probability that a damaged structure is correctly classified as damaged, see Fig. 1.

$$
P O D=1-\beta
$$

The validation technique is visualized in Fig. 9 where merely the damaged state for the decisive element is shown, that is, the element right next to the right support, so $E_{9}$. The empirical distribution (histogram in blue) aligns with the theoretical one and the POD is close to $99.4 \%$, which is the complement of required false-positive rate $\beta$. Hence, it can be concluded that the prediction based on healthy vibration was accurate. For completeness, the validation procedure is applied to all other stiffness parameters as well, but the results are not shown here. As expected, the test's response to a $5 \%$ stiffness decrease in any other parameter $E_{2}, \ldots, E_{9}$ is very strong and the non-centrality is beyond the minimum value, so $\lambda>\lambda_{\text {min }}$. That is because the minimum detectable damage in all other (non-critical) elements is below $5 \%$ and the monitoring system is tuned in a conservative manner.

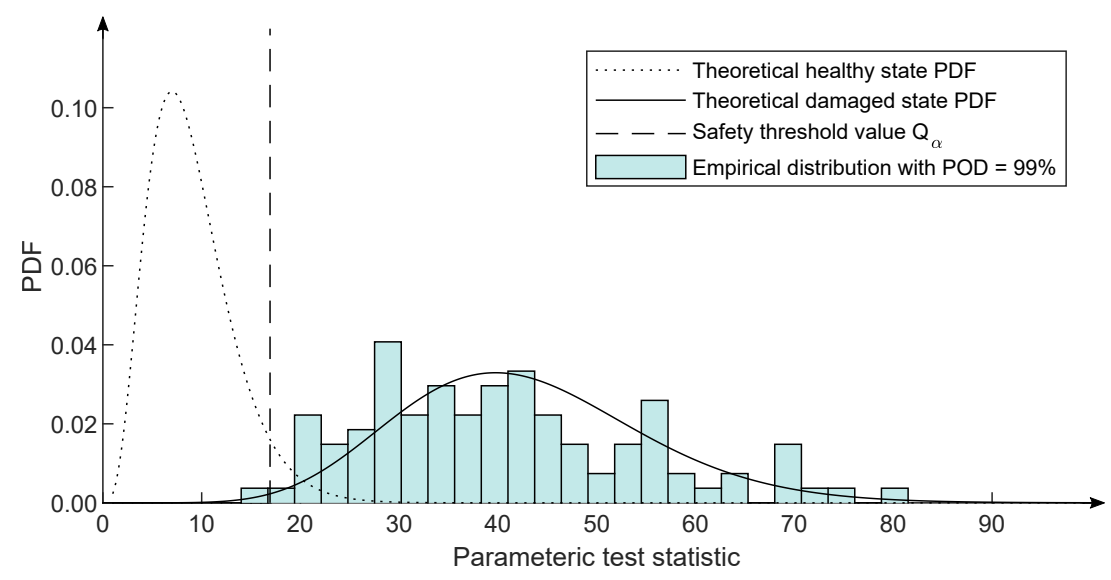

Figure 9: Empirical probability density function (PDF) and power of detection (POD) for a $5 \%$ stiffness decrease in stiffness parameter $E_{9}$. 


\section{Discussion}

The main results can be summarized as follows. The minimum measurement time of the examined beam mainly depends on the mode shapes (see Sec 4.2) and their visibility (see Sec 4.3) at the candidate sensor locations. A non-symmetrical sensor arrangement can also lead to a symmetrical distribution for the minimum measurement duration in each health parameter, and may lead to better performances than a classical symmetrical sensor placement.

Uncertainties. The Fisher information is a statistical quantity estimated based on data, and thus, subject to uncertainties. Consequently, the minimum measurement duration $T_{h}$ and the maximum measurement duration over all elements $T_{\max }$ from Eq, (17) and (18) are also scattered quantities. To quantify the uncertainties, the optimization procedure for one sensor and eight sensor locations P1-P8 was repeated twenty times with error bars shown in Fig. 7 . The standard deviation is below $10 \%$ of the total measurement duration.

Changing time lags. Optimizing the sensor configuration for a fixed number of sensors is consistent because the same input parameters can be used, but for a varying number of sensors, inconsistencies are introduced. Due to the mathematical requirement regarding the model order $\min \{p r, q r\}>2 m$, the time lag parameters $p$ and $q=p+1$ for the block Hankel matrix in Eq. (2), it is technically possible to reduce the minimum number of time lags for an increasing number of sensors $r$ because the number of modes $m$ remains the same. For eight sensors and four modes of vibration, the time lag is merely $q=3$, so 8.3 milliseconds. An undesired consequence is that the block Hankel matrix changes in size, and so does the left null space and the residuum vector which also influences all parameters derived from it (and their computation time), e.g. the Fisher information and the minimum measurement duration, see Eq. (17). A reduced number of time lags could cause that low-vibration waves do not have enough time to travel from one sensor to all others. Whether or not this is a problem remains an open question because, as shown in this paper, damage detection is also possible with a single sensor. If computational efficiency is a problem, it is generally recommended to work with the minimum time lag values $q=2 \mathrm{~m} / \mathrm{r}$.

Changing excitation. In the presented form, the optimal sensor placement depends on the excitation characteristics as well as the noise contamination. The noise contamination during training will lead to spurious modes that might dominate the structural modes (indicated through noise components in the signal with higher singular values greater than the structural ones). As a result, the singular vectors corresponding to structural modes get pushed into the null space and bias the entire framework. The noise contamination during testing could also lead to spurious modes of vibration that are not orthogonal to the null space, so the residual vector from Eq. (4) will not attain the Gaussian properties. One way to enhance the robustness towards noise is to set the model order higher than $2 m$ to create space for noise modes. Another way is to use a Gaussian residual vector that is more robust to change in the excitation characteristics, see for example [10].

Combinatorial explosion. The exhaustive search approach-where every possible sensor configuration is analyzed-worked well for the examined pin-supported beam with eight sensor locations but might be computationally burdensome for large structures with many candidate sensor locations. For example, for a 100m-long bridge with 60 possible sensor locations and 10 available monitoring channels, the number of sensor combinations increases to $N_{c}=7.5 \cdot 10^{10}$, see Eq. (21). To overcome the problem of combinatorial explosion, the performance criterion has to be combined with efficient optimization approaches, e.g. genetic algorithms, simulated annealing or others, see $[19,14]$ for an overview.

\section{Conclusions}

A distinguishing characteristic of stochastic subspace-based damage detection (SSDD) is the profound mathematical theory that accurately models the healthy and, more importantly, the damaged state. The formula for the minimum detectable damage is a central element, as it links abstract statistical quantities (e.g. the Fisher information) to practical engineering units, such as the minimum measurement duration. Due to the statistical framework, the damage detectability increases (asymptotically) with increasing sample size $N$. Vice versa, the necessary measurement duration $T=N f_{s}$ can be interpreted as a performance criterion for the damage detectability.

A great advantage of the presented performance criterion is that is can be calculated based on vibration data from the healthy structure. In fact, only one single transient analysis under white noise excitation is necessary to generate the system output at all candidate locations. Simulation in the damaged state are merely necessary to validate the method's capability of finding the optimal sensor layout. Another advantage is that both the input and output parameters are intuitive to handle. The input parameter is the desired detectable damage in percent of a stiffness parameter. It can be linked to the material utilization rate 
of the structural analysis and defined individually for each structural component. Hence, the sensor placement can be tuned to become more sensitive to damage in vital structural components or damage hot spots. The output parameter, on the other hand, is the required measurement duration. It is a function of the desired damage detectability and might soon replace the general rules of thumbs that can be found in the literature, see $[8,15]$.

\section{Acknowledgments}

The financial support from the Natural Sciences and Engineering Research Council of Canada (NSERC), the German Academic Exchange Service (DAAD) and the Mitacs Globalink Research Award is gratefully acknowledged.

\section{References}

[1] É. Balmès, M. Basseville, L. Mevel, H. Nasser, and W. Zhou. Statistical model-based damage localization: a combined subspace-based and substructuring approach. Structural Control and Health Monitoring, 15(6):857-875, 2008.

[2] M. Basseville. Information criteria for residual generation and fault detection and isolation. Automatica, 33(5):783-803, 1997.

[3] M. Basseville, M. Abdelghani, and A. Benveniste. Subspace-based fault detection algorithms for vibration monitoring. Automatica, 36(1):101-109, 2000.

[4] M. Basseville, A. Benveniste, G. Moustakides, and A. Rougee. Optimal sensor location for detecting changes in dynamical behavior. IEEE Transactions on Automatic Control, 32(12):1067-1075, 1987.

[5] M. Basseville, L. Mevel, and M. Goursat. Statistical model-based damage detection and localization: subspace-based residuals and damage-to-noise sensitivity ratios. Journal of Sound and Vibration, 275(3-5):769-794, 2004.

[6] A. Benveniste, M. Basseville, and G. Moustakides. The asymptotic local approach to change detection and model validation. IEEE Transactions on Automatic Control, 32(7):583-592, 1987.

[7] C. Boller, F.-K. Chang, and Y. Fujino. Encyclopedia of structural health monitoring. John Wiley, Chichester West Sussex U.K., 2009.

[8] R. Brincker and C. E. Ventura, editors. Introduction to Operational Modal Analysis. Wiley, New York, 2015.

[9] M. Döhler, K. Kwan, and D. Bernal. Optimal sensor placement with a statistical criterion for subspace-based damage detection. In Proceedings of the IMAC - 31st International Modal Analysis Conference, volume 4, pages 219-229, New York, 2013. Springer.

[10] M. Döhler, L. Mevel, and F. Hille. Subspace-based damage detection under changes in the ambient excitation statistics. Mechanical Systems and Signal Processing, 45(1):207-224, 2014.

[11] G. H. James, T. G. Carne, and J. P. Lauffer. The natural excitation technique (next) for modal parameter extraction from operating structures. Modal Analysis: the International Journal of Analytical and Experimental Modal Analysis, 10(4):260277, 1995.

[12] Y. Kaya, A. Mendler, and C. Ventura. Structural health monitoring network in British Columbia, Canada. In Proceedings of the EWSWH - 9th European Workshop on Structural Health Monitoring, 2018.

[13] A. Mendler, S. Allahdadian, M. Döhler, L. Mevel, and C. Ventura. Minimum detectable damage for stochastic subspacebased methods. In IOMAC - International Operational Modal Analysis Conference, 2019.

[14] W. Ostachowicz, R. Soman, and P. Malinowski. Optimization of sensor placement for structural health monitoring: a review. Structural Health Monitoring, 13(4):147592171982560, 2019.

[15] C. Rainieri and G. Fabbrocino. Operational Modal Analysis of Civil Engineering Structures. Springer, New York, 2014.

[16] M. van de Wal and B. de Jager. A review of methods for input/output selection. Automatica, 37(4):487-510, 2001.

[17] P. van Overschee and B. de Moor. Subspace identification for linear systems: theory-implementation-application. Springer Science \& Business, Berlin, 2012. 
[18] H. Wenzel. Health monitoring of bridges. Wiley, Chichester U.K., 2009.

[19] T.-H. Yi and H.-N. Li. Methodology developments in sensor placement for health monitoring of civil infrastructures. International Journal of Distributed Sensor Networks, 8(8):612726, 2012. 